\title{
Transición de fase de baja temperatura en cerámicas de PZT ricas en circonio
}

\author{
B. JIMÉNEZ ${ }^{1}$ Y J.M. VICENTE ${ }^{2}$ \\ ${ }^{1}$ Instituto de Ciencia de Materiales de Madrid. CSIC. Cantoblanco. Madrid. \\ ${ }^{2}$ Dpto. de Física. Universidad de Alcalá. Alcalá de Henares.
}

\begin{abstract}
Se han realizado medidas de dilatación tèrmica y del módulo de Young complejo en función de la temperatura en cerámicas ferroeléctricas de composiciones ricas en circonio de fórmula $\mathrm{PbZr}_{1-\mathrm{x}} \mathrm{Ti}_{\mathrm{x}} \mathrm{O}_{3}, \mathrm{x}=15.0-5.0 \%, \mathrm{y} \mathrm{Pb} \mathrm{Pb}_{1-\mathrm{y} / 2}\left(\mathrm{Zr}_{1-\mathrm{x}} \mathrm{Ti}_{\mathrm{x}}\right)_{1-\mathrm{y}} \mathrm{Nb}_{\mathrm{y}} \mathrm{O}_{3}$ con $\mathrm{x}=$ $3.5,3.0 \%, y=2.6 \%$. En la composición PZT $(95 / 5)$ se han realizado también medidas dieléctricas en función de la temperatura. Los resultados de estas medidas muestran anomalías a las temperaturas de $145^{\circ} \mathrm{C}(\mathrm{x}=15 \%), 110^{\circ} \mathrm{C}(\mathrm{x}=5 \%), 35^{\circ} \mathrm{C}(\mathrm{x}=3.5 \%) \mathrm{y}$ $28^{\circ} \mathrm{C}(\mathrm{x}=3 \%)$, respectivamente, además de las correspondientes a las transiciones de fase ferroparaeléctrica. Teniendo en cuenta el diagrama de fases, las muestras con $x<6 \%$ se encuentran en la fase antiferroeléctrica ortorrómbica y la que tiene la composición con $x=15 \%$ se encuentra en la fase ferroeléctrica con estructura romboédrica (RB) en la zona de baja temperatura. En la composición con $\mathrm{x}=5 \%$ todas las características medidas presentan anomalías a la temperatura de $110^{\circ} \mathrm{C}$ en el primer recorrido térmico subiendo la temperatura, donde se produce el cambio de fase antiferroeléctrica- ferroeléctrica con estructura romboédrica. En el recorrido de enfriamiento la fase ferroeléctrica se mantiene hasta los $28^{\circ} \mathrm{C}$, temperatura en que se pasa de nuevo a la fase antiferroeléctrica. Se produce, por tanto, una histéresis térmica muy grande. La temperatura de la transición ferro-ferroeléctrica de la composición $\mathrm{x}=15 \%$ se comporta de acuerdo con el diagrama de fases y la de $3.5 \%$ con un aditivo de $2.5 \%$ de $\mathrm{Nb}$ tiene una temperatura de transición muy alejada de la que le correspondería por el diagrama de fases.
\end{abstract}

Palabras clave: Cerámicas, ferroeléctricos, transición de fase, módulo elástico.

\section{Low temperature phase transition in zirconium-rich PZT ceramics}

Measurements of thermal expansion and complex Young's modulus in ferroelectric ceramics of zirconium-rich compositions and formula $\mathrm{PbZr}_{1-\mathrm{x}} \mathrm{Ti}_{\mathrm{x}} \mathrm{O}_{3}, \mathrm{x}=15.0-5.0 \%$, and $\mathrm{Pb}_{1-\mathrm{y} / 2}\left(\mathrm{Zr}_{1-\mathrm{x}} \mathrm{Ti}_{\mathrm{x}}\right)_{1-\mathrm{y}} \mathrm{Nb}_{\mathrm{y}} \mathrm{O}_{3}$ with $\mathrm{x}=3.5-3.0 \%, \mathrm{y}=2.6 \%$ as a function of the temperature were performed. In composition with $x=5 \%$ dielectric measurements as a function of the temperature were also done. The results of the measurements show anomalies in the studied characteristics at temperatures of $145^{\circ} \mathrm{C}(\mathrm{x}=15 \%), 110^{\circ} \mathrm{C}(\mathrm{x}=5 \%)$, $35^{\circ} \mathrm{C}(\mathrm{x}=3.5 \%)$ and $28^{\circ} \mathrm{C}(\mathrm{x}=3 \%)$, apart of those of the ferro-paraelectric phase transition. The phase diagram indicates that, at room temperature, compositions with $x<6 \%$ should be in the antiferroelectric phase with an orthorrombic structure and that with $\mathrm{x}=15 \%$ in the ferroelectric phase with rhomboedric structure. In the composition $\mathrm{x}=5 \%$ all the measurements show anomalies at $110^{\circ} \mathrm{C}$ in the first heating run, where the antiferroelectric phase changes to the ferroelectric phase of rhomboedric structure. In the cooling run the ferroelectric phase remains till $28^{\circ} \mathrm{C}$. At this temperature the antiferroelectric phase is again obtained. A large thermal hysteresis is present. The ferro- ferroelectric temperature phase transition of the composition with $x=15 \%$ behaves according to the phase diagram and that with $\mathrm{x}=3.5 \%$ is quite far away of the phase diagram values. Explanations for these results are given.

Key words: Ceramics, ferroelectrics, phase transition, elastic modulus.

\section{INTRODUCCIÓN}

Según el diagrama de fases de la solución sólida $\left(\mathrm{PbZrO}_{3}\right)_{1-\mathrm{x}}$ $-\left(\mathrm{PbTiO}_{3}\right)_{x^{\prime}}$ Figura 1, las composiciones con $\mathrm{x}<0.08$ se encuentran en una fase antiferroeléctrica, con estructura cristalina ortorrómbica (1) a temperatura ambiente. Al aumentar la temperatura, las composiciones con $\mathrm{x}<0.04$ pasan a la fase paraeléctrica, las composiciones con $0.04<\mathrm{x}<0.08$ pasan a la fase romboédrica de alta temperatura. En ambos cambios de fase existe una región morfotrópica con pequeños cambios en los valores de $\mathrm{x}$ donde coexisten las fases con estructuras ortorrómbica antiferroeléctrica $(\mathrm{AF})$ y romboédrica ferroeléctrica (F) para $\mathrm{x}>0.04$ y cúbica para $\mathrm{x}<0.03$.

La sustitución de algunos cationes del radical $\left(\mathrm{Zr}_{1-\mathrm{x}} \mathrm{Ti}_{\mathrm{x}}\right)_{1-\mathrm{y}}$ por $\mathrm{Nb}$ en la proporción y\%, lleva a una prolongación de la fase ferroeléctrica romboédrica hasta composiciones con valores de $x$ que se encontrarían en la fase ortorrómbica antiferro- eléctrica (2) y con unas temperaturas de transición muy próximas a la temperatura ambiente.

Medidas de constante dieléctrica y polarización espontánea en función de la temperatura (2-5) muestran estos cambios de fase con picos o saltos, respectivamente, en las temperaturas correspondientes a la transición de fase.

La dilatación térmica presenta anomalías en los cambios de fase estructural, (6), pero esta característica no ha sido exhaustivamente estudiada en composiciones como las que tratamos en este trabajo.

Igualmente, es conocido que en las transiciones de fase estructurales se producen acoplos entre el parámetro de orden y la deformación espontánea del material que dan lugar a claras e importantes anomalías en los parámetros elásticos, (7-9). Sin embargo, no se encuentran estudios detallados de anoma- 
lias elásticas en las transiciones $\mathrm{AF}$ / F que aporten información sobre el mecanismo que da lugar a las mismas.

En este trabajo se realiza un estudio de las transiciones citadas a través del comportamiento, en función de la temperatura, de los módulos elásticos, coeficientes de fricción interna y dilatación térmica, con el fin de aportar nueva información que permita un mejor entendimiento de las transiciones de fase estructurales en materiales ferroeléctricos.

\section{PROCEDIMIENTO EXPERIMENTAL}

Las cerámicas de Titanato Circonato de Plomo, PZT, utilizadas en este estudio, proceden del Institute of Ceramics of Shangai (composiciones con $3.5 \%$ y $3.0 \%$ de Ti y substituciones de $2.6 \%$ de $\mathrm{Nb}_{2} \mathrm{O}_{5}$ ) y del Instituto de Cerámica y Vidrio (composiciones con proporciones de 5\% y 15\% de Ti sin substituciones de $\mathrm{Nb}$ ). Las cerámicas fueron obtenidas por el procedimiento de mezcla de oxidos, calcinación, molienda y posterior sinterización a $1250^{\circ} \mathrm{C}$ en atmósfera de $\mathrm{PbO}$.

Para las medidas de dilatación térmica y módulo de Young se han empleado muestras de dimensiones $11 \times 2.5 \times .4 \mathrm{~mm}^{3}$ sobre las que se depositan electrodos de pintura de plata cuando se requieren medidas dieléctricas.

Para las medidas de dilatación térmica y módulo de Young se utilizó el equipo DMA7 en forma de análisis termomecáni-

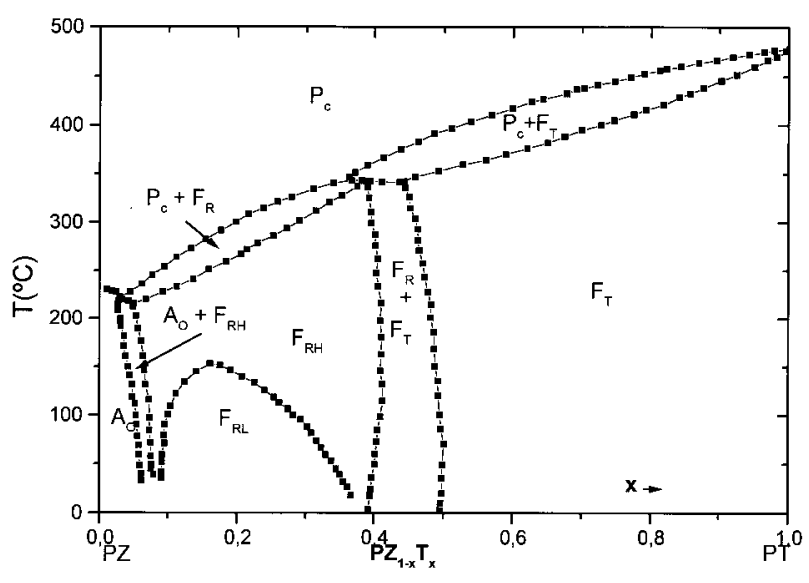

Figura 1.- Diagrama de fases del sistema (PbTiO3 )x- (PbZrO3)1-x

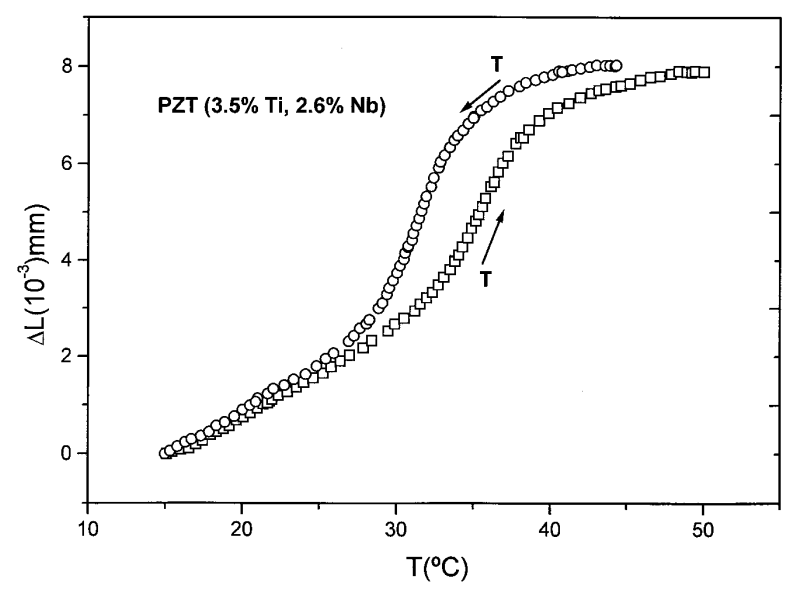

Figura 2.- Ciclo térmico de dilatación térmica de la composición $x$ $=3.5 \%$. co (TMA) y de análisis dinámico (DMA), respectivamente. El DMA se basa en la técnica de flexión por apoyo en tres puntos (TPB).

Las medidas eléctricas se realizaron en un puente de impedancias a las frecuencias de $1 \mathrm{kHz}$ y $10 \mathrm{kHz}$ con un barrido térmico de $2^{\circ} \mathrm{C} / \mathrm{min}$.

\section{RESULTADOS EXPERIMENTALES}

En las Figuras 2 y 3 se presentan las variaciones de la longitud de la muestra en función de la temperatura para las cerámicas de composición PZT(96.5/3.5) y PZT(95/5). Los resultados muestran los cambios que se producen en las transiciones de fase ferro-ferroeléctrica $\mathrm{F} / \mathrm{F}\left(\mathrm{T}=35^{\circ} \mathrm{C}\right)$, y antiferro-ferroeléctrica $\mathrm{AF} / \mathrm{F}\left(\mathrm{T}=110^{\circ} \mathrm{C}\right)$ respectivamente, así como la histéresis térmica del ciclo térmico.

Las Figuras 4 y 5 representan la variación con la temperatura del módulo del Young y de la tangente de pérdidas mecánicas para las cerámicas de la misma composición que las de las Figuras 2 y 3. Las histéresis que aparecen en los ciclos térmicos se corresponden con las obtenidas en la dilatación térmica para las mismas composiciones. Inmediatamente después de la terminación del primer ciclo térmico se hace uno nuevo y se obtienen los resultados de la Figura 6, donde aparece una fuerte subida del módulo de Young alrededor de $40^{\circ} \mathrm{C}$ y una

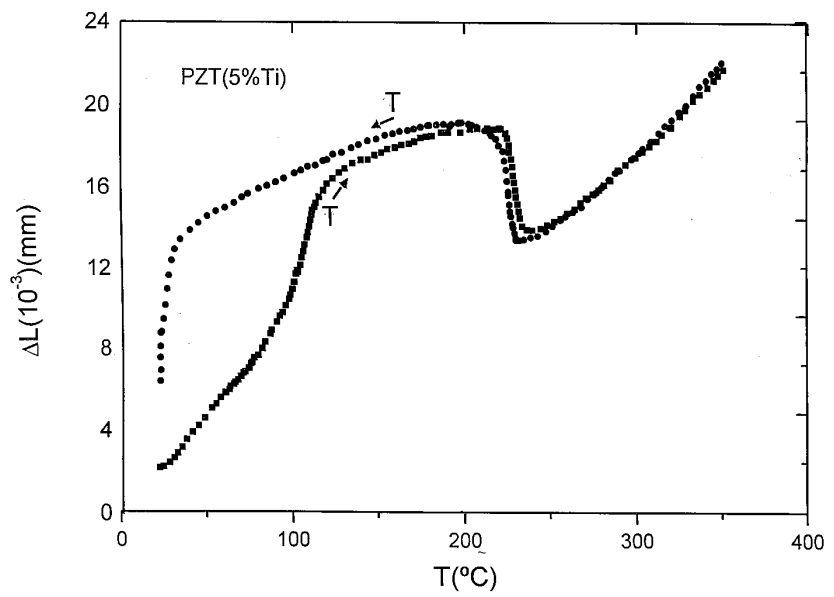

Figura 3.- Ciclo térmico de dilatación térmica de la composición $x=5 \%$.

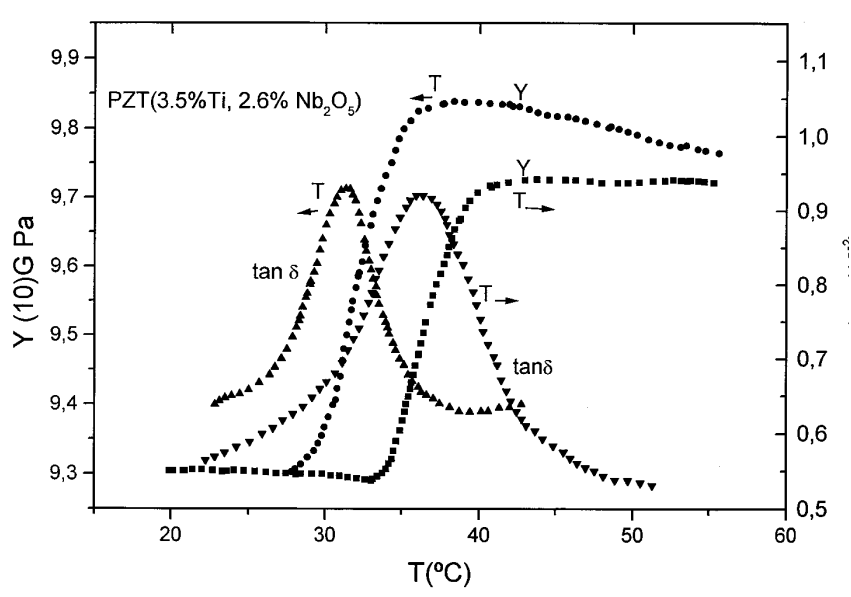

Figura 4.- Ciclo térmico del módulo de Young y tangente de pérdidas mecánicas para la muestra $\mathrm{x}=3.5 \%$. 
pequeña anomalía a los $110^{\circ} \mathrm{C}$ correspondiente a la transición $\mathrm{AF} / \mathrm{F}$. La derivada térmica del módulo de Young refleja claramente la existencia de esa anomalía.

En la Figura 7 se muestra la variación de la constante dieléctrica en función de la temperatura para la composición PZT(95/5). Se observa la anomalía en la curva de subida térmica a la temperatura de $110^{\circ} \mathrm{C}$ y la de bajada alrededor de $28^{\circ} \mathrm{C}$, con una considerable histéresis térmica.

\section{DISCUSIÓN DE RESULTADOS}

En el diagrama de fases, Figura 1, las fronteras entre dos fases cristalinas no están completamente definidas. Hay un intervalo de composiciones en las que coexisten ambas fases, es la frontera morfotrópica. En el caso que estamos tratando, hay un intervalo de un $2 \%$ de Ti a partir de la composición 98/2 hasta la 93/7 donde coexisten las fases ortorrómbica- AF y la romboédrica-F de alta temperatura. Debido a esta situación, es posible que algunos parámetros o características del material sean particularmente sensibles a alguna de las fases coexistentes, como parecen indicar los resultados obtenidos para la expansión térmica y el módulo elástico. Ambos presentan anomalías bastante definidas en ciertas temperaturas por debajo de la de transición de fase ferroparaeléctrica, F/P.

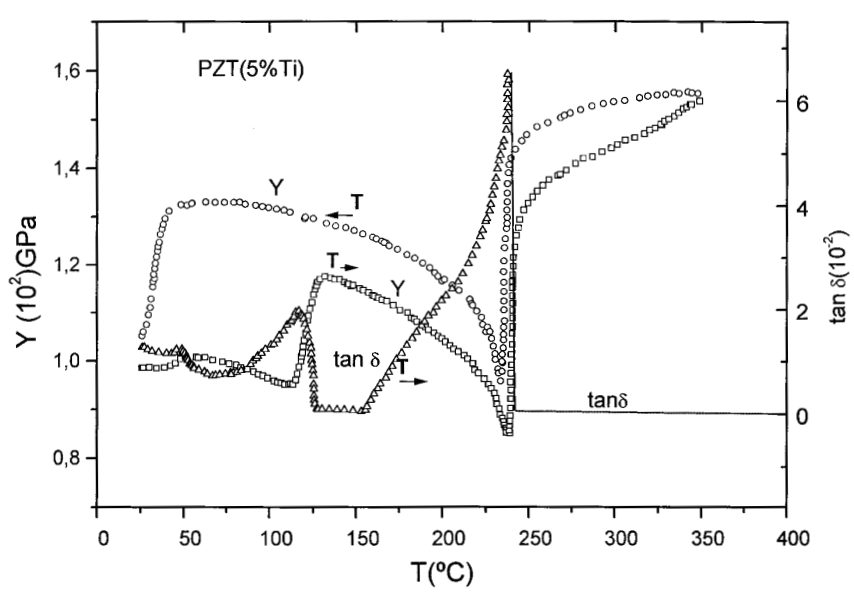

Figura 5.- Ciclo térmico del módulo de Young y tangente de pérdidas mecánicas para la muestra $\mathrm{x}=5 \%$.

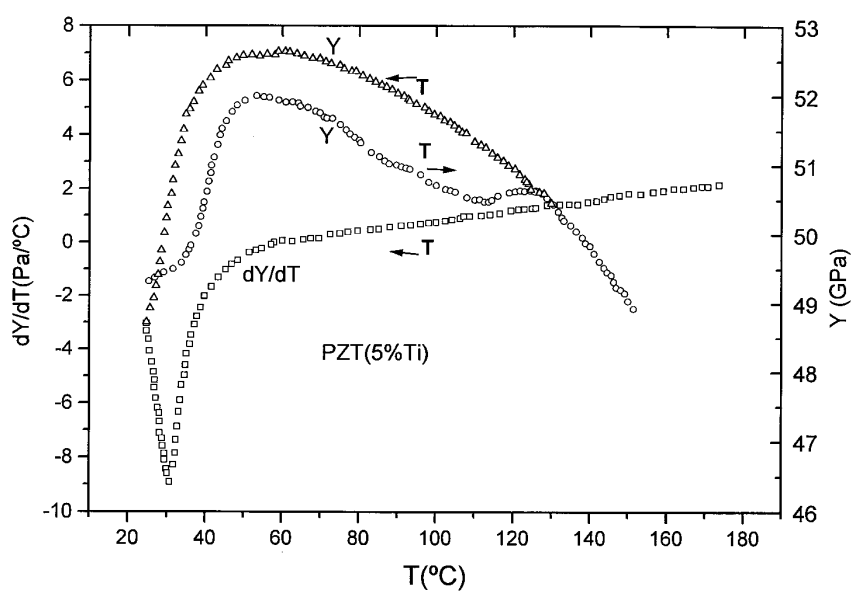

Figura 6.- Segundo ciclo térmico del módulo de Young y tangente de pérdidas mecánicas para la muestra $\mathrm{x}=5 \%$.
Estas anomalías se pueden atribuir a cambios de fase estructural entre fases ferroeléctricas, $\mathrm{F} / \mathrm{F}$, o entre una fase antiferroeléctrica y una ferroeléctrica, $\mathrm{AF} / \mathrm{F},(1,6)$.

Teniendo en cuenta los resultados de las Figuras 2 y 4 correspondientes a la composición $96.5 / 3.5(2.5 \% \mathrm{Nb})$, podemos comprobar que existe un proceso reversible con histéresis térmica, tanto para el módulo elástico como para la dilatación térmica.

En el diagrama de fases, la transición de fase AF / F para esta composición se produce justamente a esa temperatura, $215^{\circ} \mathrm{C}$. La anomalía de baja temperatura puede atribuirse a una transición entre dos fases romboédricas de alta y baja temperatura de una parte del material (6). No obstante, los estudios que se han realizado sobre muestras con diferentes proporciones de titanio $(3,6)$ a las que se les añade un pequeño porcentaje de $\mathrm{Nb}_{2} \mathrm{O}_{5}$ parecen indicar que lo que se produce es un corrimiento de la frontera de la transición $\mathrm{F} / \mathrm{F}$ hacia concentraciones más bajas y temperaturas más altas. Sin embargo, no es descartable que coexistan las fases AF y F en un estrecho intervalo de temperatura (3).

Tambien conviene destacar que en una composición pura $95 / 5, \sin \mathrm{Nb}_{2} \mathrm{O}_{5}$ añadido, se produce la transición de fase $\mathrm{AF} / \mathrm{F}$ a la temperatura indicada por el diagrama de fases $(\mathrm{T}=$ $110^{\circ} \mathrm{C}$ ) en el recorrido de temperaturas crecientes, pero no en el de temperaturas decrecientes. Aparece una histéresis térmica muy ancha, con una anomalía alrededor de $30^{\circ} \mathrm{C}$ y en un

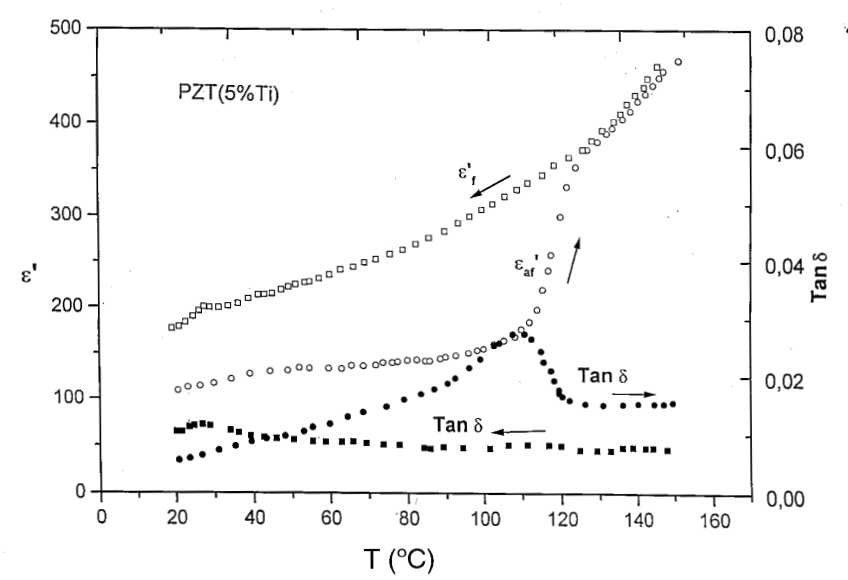

Figura 7.- Ciclo térmico para la constante dieléctrica y tangente de pérdidas dieléctricas para la muestra $\mathrm{x}=5 \%$.

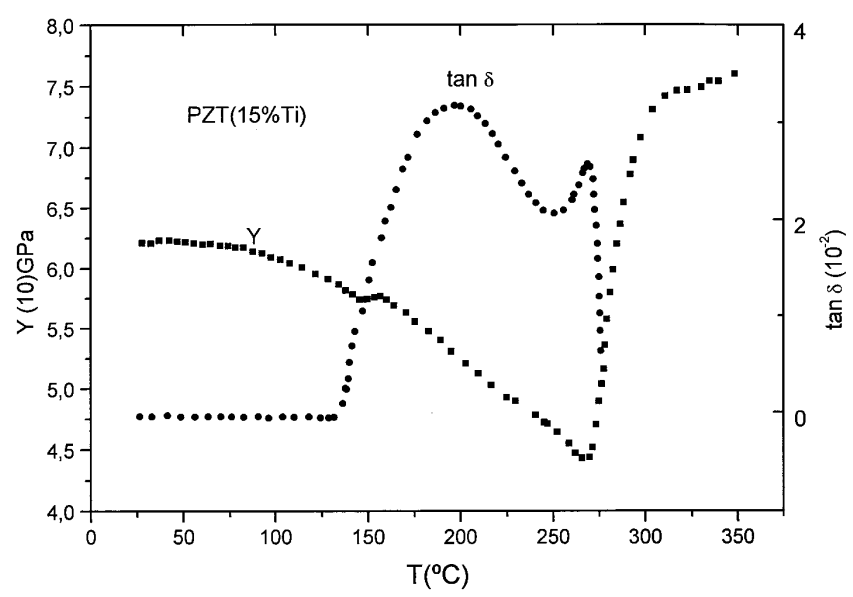

Figura 8.- Módulo de Young y tangente de pérdidas en función de la temperatura para la muestra de composición $\mathrm{x}=15 \%$ 
segundo calentamiento, realizado inmediatamente después de la bajada, la anomalía se produce a $40^{\circ} \mathrm{C}$, Figura 6 , además de la anomalía a la misma temperatura $\left(110^{\circ} \mathrm{C}\right)$ que la del primer calentamiento, aunque su escalón es bastante menor. En la segunda bajada se produce una histéresis con el mismo intervalo de temperatura. Es decir, los ciclos térmicos son de una gran histéresis térmica y se necesita un tiempo largo para recuperar el estado inicial de la muestra. Los resultados sugieren que después del primer calentamiento la muestra contiene más fase ferroeléctrica metaestable y menos antiferroeléctrica.

La transición de fase AF / F se puede tratar por consideraciones energéticas.

La energía libre de un material antiferroeléctrico tiene la forma:

$\mathrm{G}\left(\mathrm{T}, \mathrm{P}_{\mathrm{a}^{\prime}} \mathrm{P}_{\mathrm{b}}\right)=\mathrm{G}_{0}(\mathrm{~T})+\mathrm{f}\left(\mathrm{P}_{\mathrm{a}}^{2}+\mathrm{P}_{\mathrm{b}}^{2}\right)+\mathrm{g}^{\prime} \mathrm{P}_{\mathrm{a}} \mathrm{P}_{\mathrm{b}}+\mathrm{h}\left(\mathrm{P}_{\mathrm{a}}^{4}+\mathrm{P}_{\mathrm{b}}^{4}\right)+$.

Donde $\mathrm{P}_{\mathrm{a}}$ y $\mathrm{P}_{\mathrm{b}}$ representan las polarizaciones espontáneas de sentido contrario.

La correspondiente a un material ferroeléctrico es:

$$
G_{1}(T, P)=G_{01}(T)+f_{1}\left(P^{2}\right)+h_{1}\left(P^{4}\right)+\ldots
$$

Tomando términos solo hasta el segundo orden en P e igualando las energías en la temperatura de transición $\mathrm{AF} / \mathrm{F}$, podemos escribir:

$$
\begin{aligned}
& \mathrm{f}\left(\mathrm{P}_{\mathrm{a}}^{2}+\mathrm{P}_{\mathrm{b}}{ }^{2}\right)+\mathrm{g}^{\prime} \mathrm{P}_{\mathrm{a}} \mathrm{P}_{\mathrm{b}}=2 \mathrm{f}_{1} \mathrm{P}^{2} \\
& \text { Como } \mathrm{P}_{\mathrm{a}}=-\mathrm{P}_{\mathrm{b}}=\mathrm{P} \text {, resulta: } \\
& 2 \mathrm{f}^{2}-\mathrm{g}^{\prime} \mathrm{P}^{2}=2 \mathrm{f}_{1} \mathrm{P}^{2}
\end{aligned}
$$

y, por tanto:

$2 f-g^{\prime}=2 f_{1}$

Además, teniendo en cuenta que $\left(\varepsilon_{\mathrm{af}}\right)^{-1}=\mathrm{f}$ y $\left(\varepsilon_{\mathrm{f}}\right)^{-1}=\mathrm{f}_{1}$ se tiene:

$$
\mathrm{f}=1 / \varepsilon_{\mathrm{af}}=\mathrm{g}^{\prime} / 2+1 / \varepsilon_{\mathrm{f}}
$$

y como la condición de estabilidad [10] de la fase AF es que g'> 0 , la constante dieléctrica de esta fase será menor que la de la fase F. Experimentalmente se encuentra, Figura 7, que $\varepsilon_{\mathrm{af}}<\varepsilon_{\mathrm{f}}$.

$$
\begin{array}{llll}
\text { Para } & \mathrm{T}=\mathrm{T}_{\text {af }} & \left(110^{\circ} \mathrm{C}\right) & \varepsilon^{\prime}=\varepsilon_{\mathrm{f}}=300 \\
\text { Para } & \mathrm{T}<\mathrm{T}_{\text {af }} & \left(110^{\circ} \mathrm{C}\right) & \varepsilon^{\prime}=\varepsilon_{\mathrm{af}}=160
\end{array}
$$

Por otra parte, teniendo en cuenta los resultados de la dilatación térmica, Figura 3, se produce un cambio importante en las dimensiones de la muestra en la temperatura de transición de fase $\mathrm{AF} / \mathrm{F}$ a los $110^{\circ} \mathrm{C}$. Este cambio da lugar a un incremento en el acoplo lineal-cuadrático (7) entre la deformación de la red cristalina (lineal) y el cambio en el parámetro de orden (cuadrático) que produce importantes anomalías elásticas en la transición. Los resultados experimentales confirman el citado incremento en el módulo de Young y un pico en la tangente de pérdidas mecánicas en el recorrido térmico de subida, pero no en el de bajada. Teniendo en cuenta que el módulo de Young experimenta un salto negativo al pasar de la fase paraeléctrica, no polar, a la ferroeléctrica, polar, el salto positivo que se observa en la transición de fase $\mathrm{AF} / \mathrm{F}$ sugiere que el acoplo es más fuerte en la fase AF que en la F, Figura 5.

Así pues, el término del desarrollo de la energía libre que tiene en cuenta el acoplo lineal-cuadrático se puede escribir:

$$
\mathrm{G}_{\mathrm{ac}}+\Delta \mathrm{G}_{\mathrm{ac}}=\mathrm{q}(\mathrm{x}+\Delta \mathrm{x}) \mathrm{P}^{2}
$$

Siendo $\mathrm{G}_{\mathrm{ac}}$ el término de la energía libre debido al acoplo en la transición de fase ferroparaeléctrica $(\mathrm{F} / \mathrm{P})$ y $\Delta \mathrm{G}_{\mathrm{ac}}$ correspondiente a la $\mathrm{AF} / \mathrm{F}$ que vendrá dado por

$$
\Delta \mathrm{G}_{\mathrm{ac}}=\mathrm{q} \Delta \mathrm{xP} \mathrm{P}^{2}
$$

De acuerdo con los resultados de la dilatación térmica, el material experimenta un salto positivo en sus dimensiones en la transición de fase AF/F como ocurre en la transición de fase F/P. Así pues, el incremento del módulo de Young en la transición AF/F tendrá el mismo sentido que en la F/P. Experimentalmente, se encuentra este comportamiento, Figura 5.

Cuando se trata de una composición que se encuentra claramente en la fase romboédrica, caso de la $85 / 15$, no se produce la anomalía de baja temperatura; se encuentran las correspondientes a la transición $\mathrm{F} / \mathrm{F}$ y a la $\mathrm{F} / \mathrm{P}$ con pequeñas histéresis térmicas en ambas transiciones y siempre reversibles, Figura 8.

En el caso de la composición $x=3.5 \%$ con un $2,6 \% \mathrm{Nb}$, los resultados muestran las anomalías en las características medidas alrededor de $35^{\circ} \mathrm{C}$ que no se corresponde con la temperatura de transición $\mathrm{AF} / \mathrm{F}$ que indica el diagrama de fases $(\approx$ $150^{\circ} \mathrm{C}$ ). Este comportamiento se ha atribuido [6] a que la introducción de esas impurezas produce desplazamintos de los iones de $\mathrm{Zr}$ y Ti, así como pequeñas rotaciones de los octaedros de oxígenos. No obstante, de acuerdo con el diagrama de fases, es posible que coexista una pequeña proporción de fase AF capaz de producir ligeras anomalías como la que aparece a temperaturas próximas a $120^{\circ} \mathrm{C}$, Figura 9 .

\section{CONCLUSIONES}

Se ha puesto en evidencia que las medidas del módulo de Young y tangente de pérdidas mecánicas proporcionan información muy importante sobre la existencia de fases antiferroeléctricas y las anomalías que se producen en la transición $\mathrm{AF} / \mathrm{F}$, que son dificilmente observables por otros procedimientos de medida de propiedades macroscópicas.

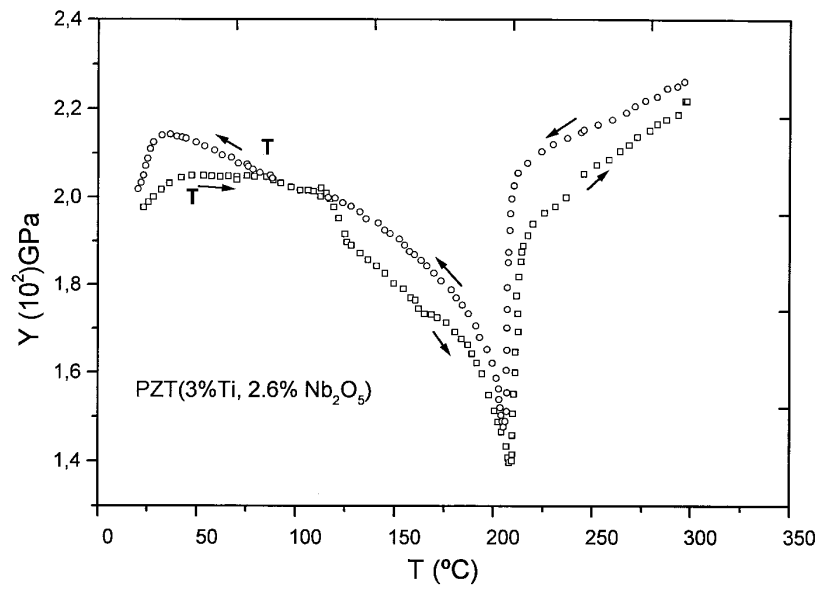

Figura 9.- Ciclo térmico del módulo de Young para la muestra de composición $\mathrm{x}=3 \%$. 
En los procesos de medida de propiedades en función de la temperatura se producen grandes histéresis térmicas, $\Delta \mathrm{T}=$ $80^{\circ} \mathrm{C}$, en las que la fase ferroeléctrica se mantiene en un estado metaestable hasta temperaturas próximas a la temperatura ambiente.

Parece comprobarse que, incluso las composiciones que han salido de la fase $\mathrm{AF}(\mathrm{Ti}=3.0 \%)$ por la incorporación de dopantes mantienen restos de fase AF.

\section{AGRADECIMIENTOS}

Al Dr. C. Moure del ICV por facilitar las muestras que hemos utilizado en este trabajo. El trabajo ha sido realizado con el soporte de los proyectos CICYT MAT 97-0711 y CICYT MAT 98-1068.

\section{BIBLIOGRAFÍA}

1. A Barbulescu, E. Barbulescu and D.Barb. "Phase Transition in PZT Solid Solutions". Ferroelectrics, 47, 221-330, (1983).
2. H.H. Berlincourt, A. Kruger and B. Jaffe. "Stability of phases in modifiedlead zirconatewith variation in pressure, electric field, temperature and composition". J. Phys. Chem. Solids. 25, 659-674, (1964).

3. Wang Yongling, L. Shengwei and Y. Qiugri. "The coexistence of AF and F Phases in PZT 95/5 Type Ceramics". Procee. First China- USA Seminar. Shangai, May, pag. 503-505, (1983).

4. Xunhy Dai and Wang Yongling. " Study of the order of the Fr (LT)- Fr(HT) Phase - transition of PZT ceramics". Phys. State Solid (a).124, 435 - 450, (1991).

5. X.L. Zhang, Z.X. Chen, L.E. Cross and W.A. Schulze. “Dielectric and piezoelectric properties of modified lead titanate-zirconate ceramics from 4.2 to 300K". J. Mat. Science, 18, 968 (1983).

6. B. Noheda. "Transiciones de fase en perovskitas ferroeléctricas puras y mixtas: PZT rico en circonio". Tesis Doctoral. Universidad Autónoma de Madrid (UAM). 1996.

7. W. Rehwald. "The study of structural phase transitions by ultrasonic experiments".

Adv. Phys. 22, 721- 755, (1973).

8. B. Jiménez and J.M. Vicente. "The low-frequency Young modulus and internal friction in $\mathrm{Pb}-\mathrm{Ca}$ and $\mathrm{Pb}-\mathrm{Zr}$ titanate ceramics". J. Phys. D: Appl. Phys. 31, 130-136, (1998).

9. A.V. Kityk, W. Schranz, A. Fuith, D. Havlik, V.P.Sopronynk and H.Warhareek. "Acoustc dispersion of $\left(\mathrm{NH}_{3} \mathrm{C}_{2} \mathrm{H}_{5}\right)_{2} \mathrm{MnCl}_{4}$ near the structural phase transition at 226K". Phys. Rev. B. 53, 3055-3060, (1996).

10. R. Blink and B. Zeks. "Soft modes in Ferroelectrics and Antiferroelectrics". North Holland Publ. Comp. Amnsterdam. 1974. 